\title{
Overview of PHENIX Physics at Forward and Backward Rapidity
}

\section{Richard Seto* for the PHENIX Collaboration}

University of California, Riverside

E-mail: richard.setoducr.edu

\begin{abstract}
Physics at forward and backward rapidity in hadron+hadron and nucleus+nucleus collisions give access to kinematic regions that give unique information about nuclear effects from cold nuclear matter, crucial in understanding signals from the final state, such as the Quark Gluon Plasma. This proceedings will focus on three areas of interest. ${ }^{\dagger}$ The first will describe a measurement of $\mathrm{J} / \psi$ suppression in $\mathrm{U}+\mathrm{U}$ collisions (measured by PHENIX in the forward and backward regions) where signs of regeneration from $c \bar{c}$ coalescence are seen. The second will include a measurement and discussion of the effects causing suppression of the $\psi(2 \mathrm{~s})$ to $\psi(1 \mathrm{~s})$ in cold nuclear matter. Lastly, I will provide an analysis update data taken with a recently added detector to the PHENIX experiment, the MPC-EX that has as its purpose, the measurement of $\pi^{0} \mathrm{~s}$ and direct photons in cold nuclear matter at low-x.
\end{abstract}

12th International Workshop on High-pT Physics in the RHIC/LHC Era 2-5 October, 2017

University of Bergen, Bergen, Norway

\footnotetext{
* Speaker.

${ }^{\dagger}$ Not all topics covered in the talk are discussed in this contribution.
} 


\section{Introduction}

These conference proceedings present some recent PHENIX results from forward and backward rapidities. It will cover several topics. The first is the nuclear modification of the $\mathrm{J} / \psi$ meson in $\mathrm{U}+\mathrm{U}$ collisions (measured by PHENIX at forward and backward rapidities) in a search for regeneration via $c \bar{c}$ coalescence at RHIC. The second is the ratio of the yields of the $\psi(2 S)$ to the $\psi(1 S)$ in $\mathrm{d}+\mathrm{Au}$ collisions to study initial and final state effects in charmonium production. Finally I will end by describing the progress on the analysis of data taken with the recently completed MPC-EX detector that has as its goal, the measurement of $\pi^{0} \mathrm{~s}$ and direct photons at forward and backward rapidity.

\section{2. $\mathbf{J} / \psi$ suppression in $\mathbf{U}+\mathbf{U}$ collisions.}

The suppression of the $\mathrm{J} / \psi$ from screening in $\mathrm{Au}+\mathrm{Au}$ collisions relative to $\mathrm{p}+\mathrm{p}$ collisions has been regarded as an important signature for the formation of the Quark Gluon Plasma(QGP). However, suppression can be from other causes. These can be initial state effects, such as the modification of parton densities and multiple scattering, or final state effects. An important effect that acts to enhance the production of the $\mathrm{J} / \psi$ in heavy ion collisions is its regeneration via coalescence, thereby countering the suppression from the screening in the QGP. This will occur if the density of charm pairs is high enough. Of course such a process is itself a signature of QGP formation that should be observable as the energy is increased resulting in more hard processes producing charm pairs. The nuclear modification factor, $R_{A A}=\frac{1}{\left\langle N_{c o l l}\right\rangle} \frac{d N^{A A} / d y}{d N^{p p} / d y}$, appears to be at a minimum at the highest RHIC energy of $200 \mathrm{GeV}$ center of mass, increasing both as one goes to lower energies, and as one goes to higher energies at $2.76 \mathrm{TeV}$ [5]. In 2012, a U+U data set where the energy density is higher than in Au+Au collisions by about 20\% [6], was taken at center of mass energy $193 \mathrm{GeV}$. The $\mathrm{R}_{A A}$ of the $\mathrm{J} / \psi$ was measured at forward and backward rapidity.

Figure 1 shows a comparison of $R_{A A}$ for $\mathrm{U}+\mathrm{U}$ and $\mathrm{Au}+\mathrm{Au}$ collisions[1]. $R_{A A}$ is seen to increase when one goes from $\mathrm{Au}+\mathrm{Au}$ to $\mathrm{U}+\mathrm{U}$ collisions. We define a relative nuclear modification factor as

$$
R_{\text {AuAu }}^{U U}=\frac{d N^{U U} / d y}{d N^{A u A u} / d y}\left(\frac{N_{\text {coll }}^{\text {AuA }}}{0.964 N_{\text {coll }}^{U U}}\right)^{2}
$$

where the $N_{\text {coll }}^{2}$ accounts for the scaling of charm coalescence, and the 0.964 accounts for the difference in the center of mass energy in $\mathrm{U}+\mathrm{U}$ vs $\mathrm{Au}+\mathrm{Au}$ collisions. If the production in central collisions was entirely due to coalescence and cold nuclear matter effects are the same, this factor should be $\sim 1$ as is seen in Figure 2. The two sets refers to two different parameterizations of the deformed Woods-Saxon distributions for the Uranium nucleus. Set 2[7] has a somewhat more compact core than the more conventional one from Matui[8], due to a somewhat smaller diffuseness parameter. (Note that Figure 1 uses set 1. Refer to [1] for the figure corresponding to set 2).

\section{3. $\psi(2 s)$ to $\psi(1 s)$ in cold nuclear matter}

$p(d)+A u$ collisions are typically used to isolate initial state effects, however, there may be final state effects at work even in these collisions since $p(d)+A$ collisions may produce a high density 


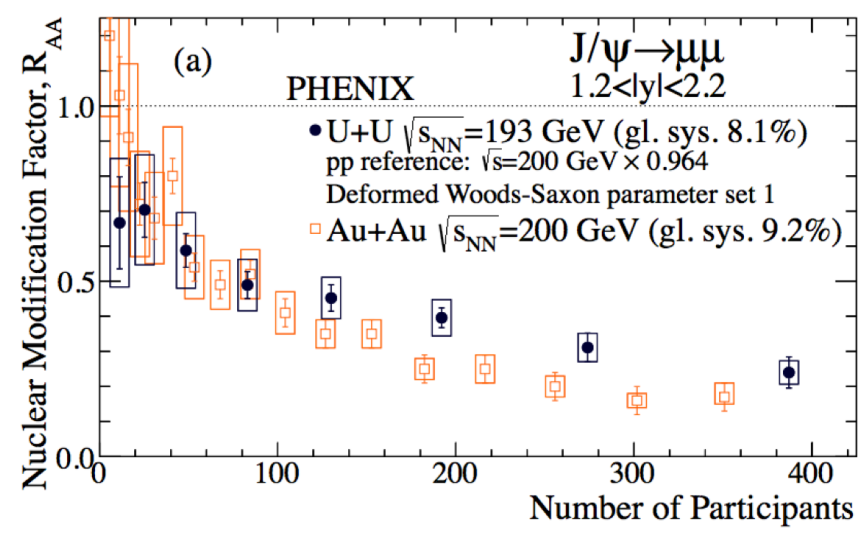

Figure 1: The nuclear modification factor, $R_{A A}$, for the $\mathrm{J} / \psi$ in $\mathrm{U}+\mathrm{U}$ (closed circles), and $\mathrm{Au}+\mathrm{Au}$ (open circles) collisions. The $\mathrm{p}+\mathrm{p}$ reference for $\mathrm{U}+\mathrm{U}$ has been scaled by a factor of 0.964 to account for the difference in cross section at $193 \mathrm{GeV}$ center of mass.

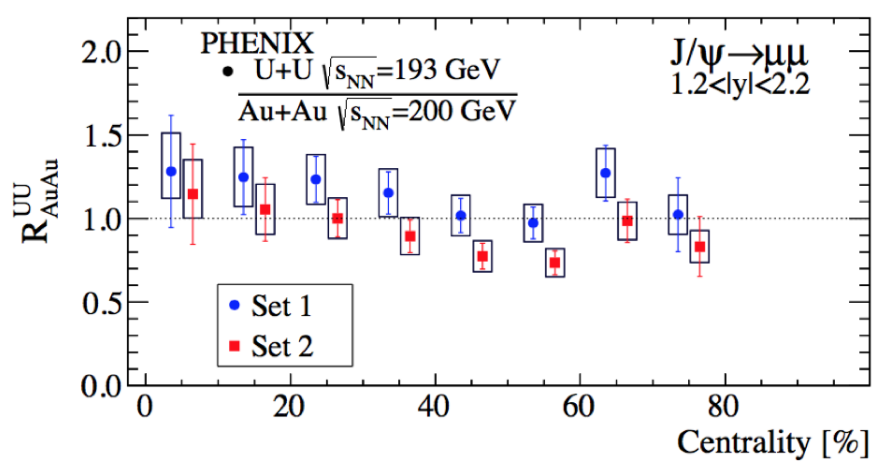

Figure 2: The relative nuclear modification factor for $\mathrm{U}+\mathrm{U}$ and $\mathrm{Au}+\mathrm{Au}$ as described in the text. The data points for the two Woods Saxon parameter sets are offset for clarity.

of particles that may induce final state interactions. One way to separate initial state effects from final state effects is to look at the ratio of the $\psi(2 \mathrm{~s})$ to $\psi(1 \mathrm{~s})$ yields, since the formation of both are thought to come from identical $c \bar{c}$ precursor states. Initial state effects on this precursor state, such as the modification of the parton distribution functions or energy loss in the nucleus, will affect the $\psi(2 \mathrm{~s})$ and $\psi(1 \mathrm{~s})$ equally. The precursor state will project out to the final state after a charmonium formation time of about $0.15 \mathrm{fm} / \mathrm{c}$. The binding energies of the $\psi(2 \mathrm{~s})(\sim 50 \mathrm{MeV})$ is much smaller than for the $\psi(1 \mathrm{~s})(640 \mathrm{MeV})$, hence final state effects will affect the $\psi(2 \mathrm{~s})$, much more strongly. These effects can by quantified by studying the double ratio of cross sections of $\psi(2 \mathrm{~s})$ to $\psi(1 \mathrm{~s})$ in $\mathrm{p}+\mathrm{Au}$ as compared with $\mathrm{p}+\mathrm{p}$ collisions. PHENIX has measured the ratio of yields of $\psi(2 \mathrm{~s})$ to $\psi(1 \mathrm{~s})$ in $\mathrm{p}+\mathrm{p}, \mathrm{p}+\mathrm{Al}, \mathrm{p}+\mathrm{Au}$, and ${ }^{3} \mathrm{He}+\mathrm{Au}$ collisions. Figure 3 shows the double ratio

$$
\left[\frac{\sigma_{\psi(2 s)}}{\sigma_{\psi(1 s)}}\right]^{p /{ }^{\beta} H e / d+A} /\left[\frac{\sigma_{\psi(2 s)}}{\sigma_{\psi(1 s)}}\right]^{p+p}
$$

as a function of rapidity[2]. At forward rapidity this double ratio is consistent with unity, which implies that final state effects are minimal. At backward rapidity the double ratio in all systems 
are at about 0.5. A major difference between the environment in the backward rapidity region as compared with the forward rapidity region, are co-moving particles that can interact and break up the more loosely bound $\psi(2 \mathrm{~s})$. Figure 3 also shows a comover model[4] that shows the correct trend, however it under-predicts the relative suppression. In order to better understand the relationship with the comover density, Figure 4 shows the PHENIX data together with data from the LHC[9][10] as a function of the comover density. The comover density is defined as the $\mathrm{dN} / \mathrm{d} \eta$ from AMPT evaluated over the same rapidity as the charmonia measurement, divided by the nuclear overlap $\left\langle S_{T}\right\rangle$ as calculated from a Glauber Monte Carlo simulation. The figure shows that the suppression of the double ratio decreases as the comover density increases, which is qualitatively consistent with the interpretation that the effect is due to $\psi(2 s)$ breakup via interactions with comovers, i.e. a final state effect.

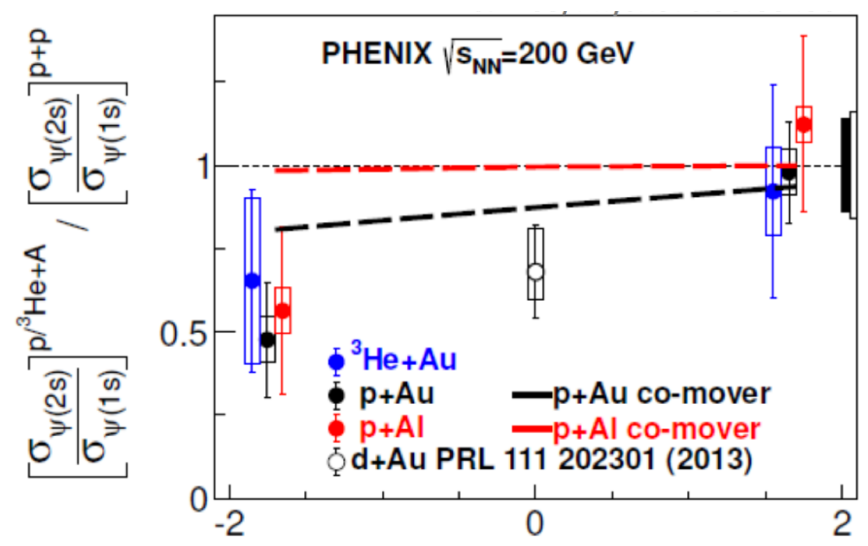

Figure 3: Double ratio of $\psi(2 \mathrm{~s})$ to $\psi(1 \mathrm{~s})$ between $\mathrm{p} /{ }^{\beta} \mathrm{He}+\mathrm{A}$ and $\mathrm{p}+\mathrm{p}$ collisions as a function of rapidity at $\sqrt{s_{N N}}=200 \mathrm{GeV}$. Also shown are comover models from [4].

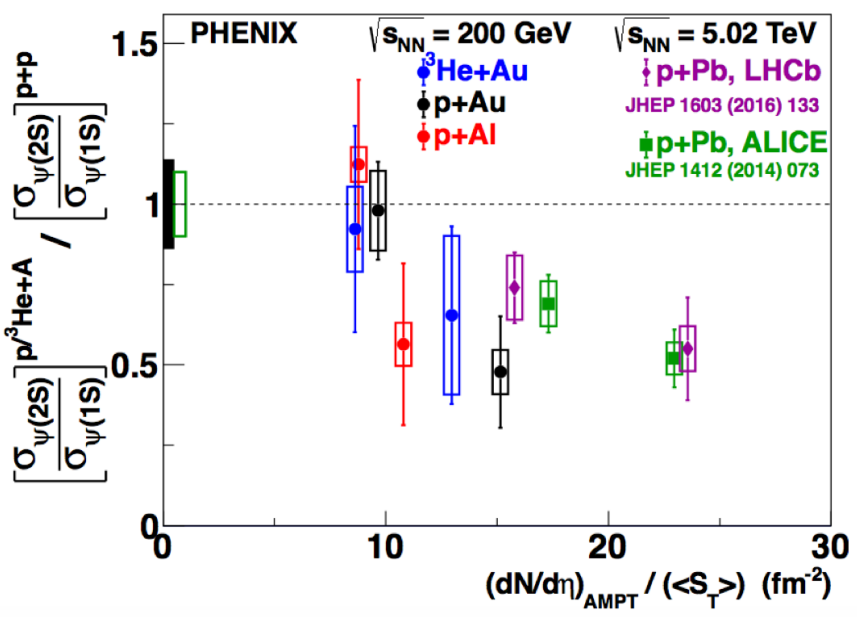

Figure 4: Double ratio of $\psi(2 \mathrm{~s})$ to $\psi(1 \mathrm{~s})$ between $\mathrm{p} /{ }^{3} \mathrm{He}+\mathrm{A}$ and $\mathrm{p}+\mathrm{p}$ collisions and including data from the LHC as a function of comover density. 


\section{The PHENIX MPC-EX detector}

The initial state for the formation of the Quark Gluon Plasma is primarily the low-x gluon distribution in the nucleus. The gluon distribution functions are not well known at low-x. Direct photons are an excellent way to measure these distributions, since they originate from the primary vertex in a simple 2 to 2 process and have no fragmentation function. To measure such photons, it is necessary to measure $\pi^{0} \mathrm{~s}$ at low-x in the forward region at RHIC. The MPC-EX (Muon Piston Calorimeter EXtenesion) is a preshower detector for the PHENIX MPC lead tungstate calorimeters that covers $3.1<|\eta|<3.8[3]$. Its purpose was to extend the capability of the calorimeter to measure $\pi^{0} \mathrm{~s}$ to high $\mathrm{p}_{T}$. The geometry of PHENIX makes it necessary that the calorimeter be placed near the vertex, hence photon showers from high $\mathrm{p}_{T}(>2 \mathrm{GeV} / \mathrm{c}) \pi^{0} \mathrm{~s}$ overlap in the MPC. The addition of the MPC-EX allows for the reconstruction of $\pi^{0} \mathrm{~s}$ even if the photon showers merge in the calorimeter. The MPC-EX is a silicon tungsten sandwich, where the detector elements in the silicon have high granularity (Figure 5). The direction of electron tracks emanating from photons that convert in the tungsten layers and are detected in the silicon and give an accurate measurement of the opening angle of the two decay photons. This, together with the total energy measured by a sum of energy measurements in the MPC-EX and MPC, and a shower energy asymmetry given by the entry point of the electrons in the MPC and the shower topology, allow for the reconstruction of $\pi^{0}$ s where only the single combined shower is detected in the MPC. This extends the range of $\pi^{0}$ mass reconstruction to the luminosity limit in the forward direction $(\sim 80 \mathrm{GeV})$. A schematic of this shown in Figure 6(bottom). Figure 7 shows the spacial distribution of hits in the MPC-EX for a single shower, and a preliminary reconstructed $\pi^{0}$ mass spectrum.

The status of the MPC-EX is as follows: The d+Au data taking run using the MPC-EX at 200, 62,39 , and $20 \mathrm{GeV}$ center of mass was in 2016. Calibrations are in their final stages. A preliminary $\pi^{0}$ spectrum and $R_{C P}$ from d+Au collisions should be completed in a few months. We estimate that the direct photon measurement will be available a year after that.

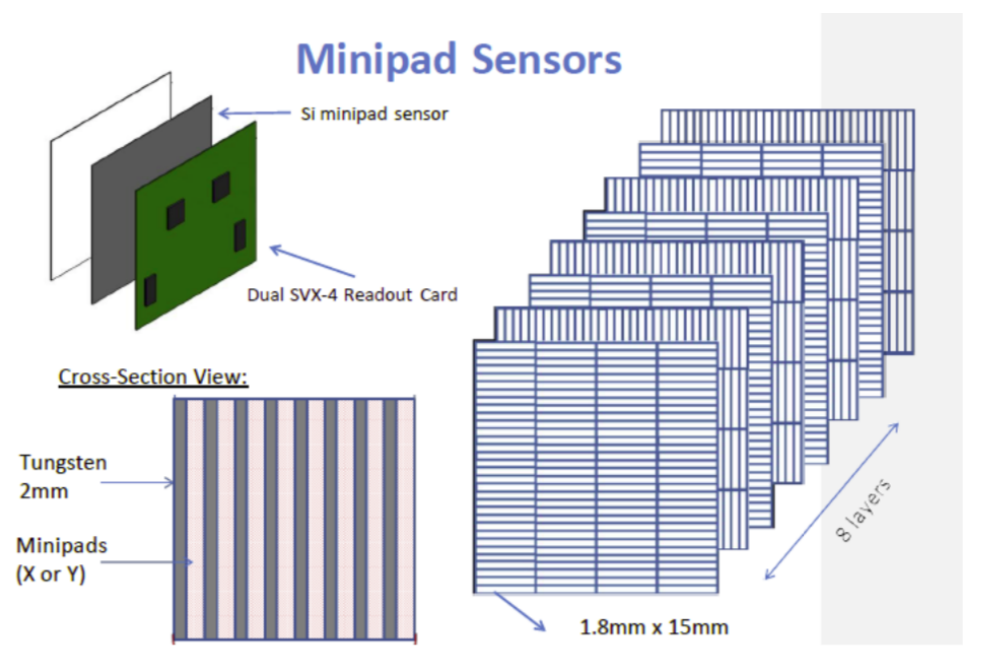

Figure 5: The structure of one module of the MPC-EX. Shown is the silicon-tungsten sandwich, together with the minipads that make up the segmentation of the detector. 

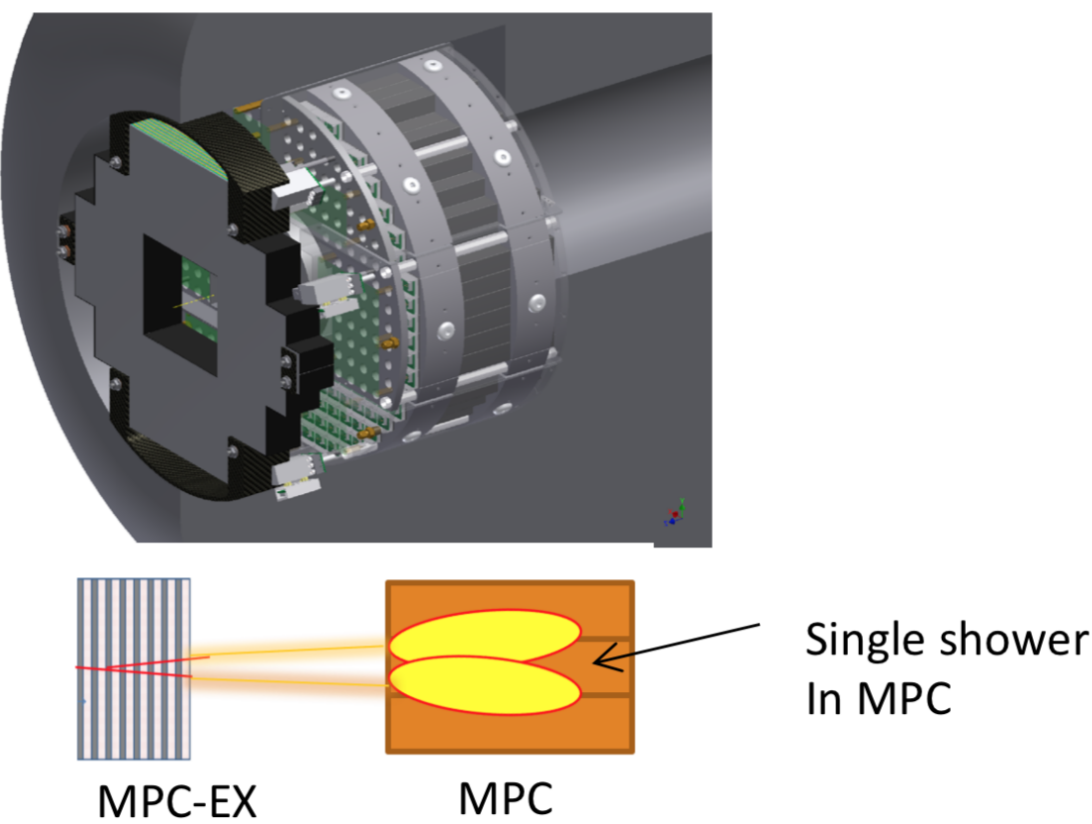

Figure 6: Top: The MPC-EX detector in black, shown in front of the MPC calorimeter. The radial coverage of the device is about $18 \mathrm{~cm}$. Bottom: schematic of a $\pi^{0}$, in which the two decay photons appear as one shower in the MPC. The tracking in the MPC-EX allows for an accurate measurement of the opening angle.

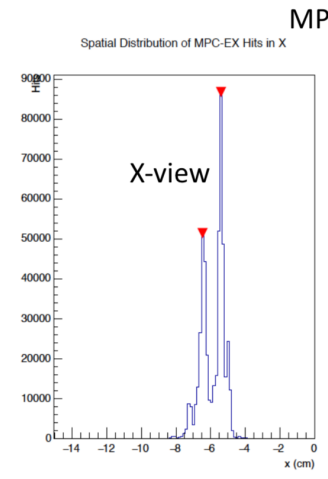
MPC-EX
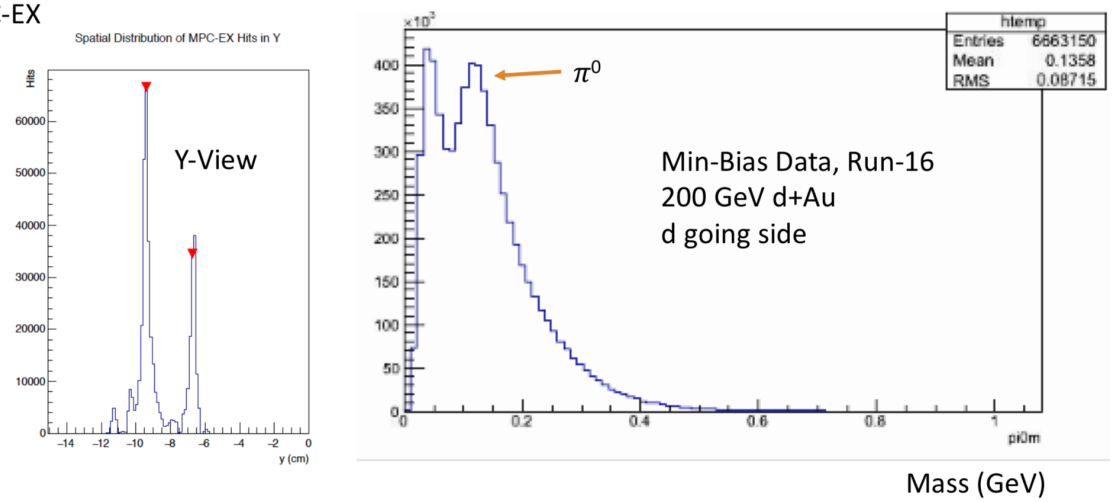

Figure 7: Left two panels: Spacial distribution of hits in the MPC-EX. The two tracks from the $\pi^{0}$ decay photons found by the reconstructiion are marked with red arrows. Right panel: Single shower $\pi^{0} \mathrm{~s}$ mass spectrum reconstructed by the MPC-EX+MPC from minimum Bias d+Au data.

\section{Conclusion}

These proceedings have covered several topics. First, a measurement of $\mathrm{J} / \psi$ suppression in $\mathrm{U}+\mathrm{U}$ collisions has shown regeneration due to the coalescence of $c \bar{c}$ pairs due to the fact that the energy density is about $20 \%$ higher than in Au+Au collisions, Secondly, a measurement of of the $\psi(2 \mathrm{~s})$ to the $\psi(1 \mathrm{~s})$ indicates that at backward rapidity where the comoving particle density is higher, the $\psi(2 \mathrm{~s})$ is suppressed by a factor of about 2 . This probably indicates final state interactions are responsible for the break up of $\psi(2 \mathrm{~s})$. Finally, thess proceedings have given an overview and status 
of the MPC-EX detector, where we hope to have first results in the next several months.

\section{References}

[1] A. Adare et al. [PHENIX Collaboration], Phys. Rev. C 93, no. 3, 034903 (2016).

[2] A. Adare et al. [PHENIX Collaboration], Phys. Rev. C 95 (2017) no.3, 034904.

[3] S. Campbell et al. [PHENIX Collaboration], arXiv:1301.1096 [nucl-ex].

[4] G. Ferreiro (2016) Private communication.

[5] J. Adam et al. [ALICE Collaboration], JHEP 1605, 179 (2016).

[6] D. Kikola, G. Odyniec and R. Vogt, Phys. Rev. C 84, 054907 (2011).

[7] Q. Y. Shou, Y. G. Ma, P. Sorensen, A. H. Tang, F. VidebÃęk and H. Wang, Phys. Lett. B 749, 215 (2015).

[8] H. Masui, B. Mohanty and N. Xu, Phys. Lett. B 679, 440 (2009).

[9] R. Aaij et al. [LHCb Collaboration], JHEP 1603, 133 (2016).

[10] B. B. Abelev et al. [ALICE Collaboration], JHEP 1412, 073 (2014). 